\title{
Control Technology for the VOCs
}

\section{Chuhong Zhan}

School of Electric Power Engineering, North China Electric Power University, Baoding 071000, China.

1941973542@qq.com

Keywords: Volatile Organic Chemicals (VOCs); Smoke Exhaust Ventilator; Photo-catalytic-oxidation Technology; TiO2 Catalyst;

\begin{abstract}
Based on extensive usage of the smoke exhaust ventilator, this paper develops a processing technology of VOCs (volatile organic compound) by photo-catalytic-oxidation technology. This method couples with the existing the smoke exhaust ventilator technology to control the cooking fume emission ---VOCs.
\end{abstract}

\section{Introduction}

Cooking fume is the oil smoke produced from acute chemical changes when we fry, stir-fry, and boil the food with the cooking oil. ${ }^{[1]}$ Cooking fume's composition is complexed and mainly include fatty acid, alkane, olefin and short-chain aldehydes after oxicracking, ketone, alcohol, ester, aromatic compound and heterocyclic compound. Most of these are toxic chemicals and damage human's respiratory tract. What's more, untreated cooking fumes from millions and millions directly emit and accumulate in atmosphere. Thus, it became a major emission source of the smog of the city. Abdullahi $\mathrm{K} \mathrm{L}^{[4]}$ and others even believe that it is the third culprit following the industrial emission and motor vehicle exhaust emission. Hence, the control of the VOCs becomes problems demanding prompt solutions.

Currently, most common smoke exhaust ventilators in market only realize the spatial transference. Most environment-friendly smoke exhaust ventilators make a good use of pressure difference to take out the cooking fumes and then separate the oil and smoke with the centrifugal function by impeller revolving. This paper plans to couple the existing smoke exhaust ventilator with the photo-catalytic oxidation to purify the gas fumes which provide the experience for the revolution of the environment-friendly smoke exhaust ventilators.

\section{Mechanism of VOCs Photocatalytic Degradation}

Photo-catalyst Nano materials like $\mathrm{TiO}_{2}$ will oxidize the VOCs into $\mathrm{CO}_{2}$ and water under the UV-irradiation.

$\mathrm{TiO}_{2}$ has stable chemical properties, non-toxic, insoluble and cheap characters. As the representative of then-type semiconductor, Nano- $\mathrm{TiO}_{2}$ 's special energy band structure makes it occupy an important position in the light-catalyzed reaction. The energy band of $\mathrm{TiO}_{2} \mathrm{xould}$ be divided into three bands: Conduction Band (CB), Forbidden Band (FB), Valence Band VB). The three bands play a key part in the light-catalyzed reaction of $\mathrm{TiO}_{2}$. This reaction includes four processes. The specific reaction mechanism of $\mathrm{TiO}_{2}$ photo-catalysis will be shown as Figure 1. ${ }^{[5]}$ 


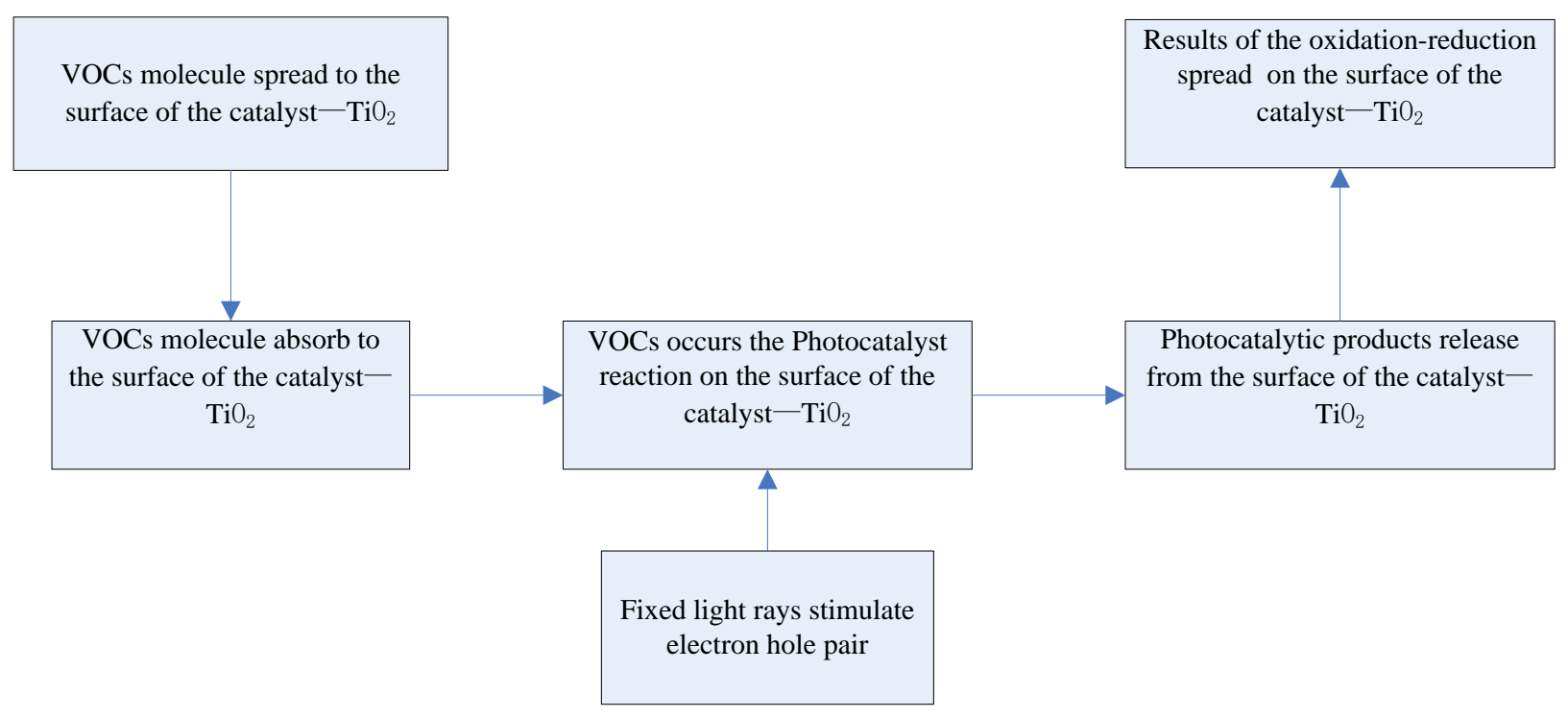

Figure 1 Steps of the photo-catalysis

Adsorption Process. VOCs, taking part in the light-catalyzed reaction, create enough material and space condition by absorbing the surface of the photo-catalyst-- $\mathrm{TiO}_{2}$. $\mathrm{TiO}_{2}$, honeycomb type, could reinforce the reaction area, especially in the Nano-level.

Stimulation by the Light. Receiving certain energetic light could stimulate electron hole pair. It is calculated that wavelength of the irradiation beam must be equal or less than $387.5 \mathrm{~nm}$. Hence, the UV-light is adopted as the light source.

Formation and Transition of the Electron Hole Pair. The electrons filled the VB will transit and form photoproduct holes on the bands when the electrons are stimulate by enough energetic highlights.

Degradation of the Organic Chemicals. Active free radicals from the light-catalyzed reaction react with the VOCs and exercise oxygenolysis. Finally, VOCs will degrade into simple inorganic matter, including $\mathrm{CO}_{2}$ and $\mathrm{H}_{2} \mathrm{O}$.

\section{The Effect of Photo-catalysis}

According to the research made by Stevens ${ }^{[5][6][7]}$ and others, with $265 \mathrm{~nm}$ light source and under the catalytic conversion of $\mathrm{TiO}_{2}$, the transformation rate of the toxic matters including methanol, acetonylbenzene, methylbenzene and PCE will be over 80\%.

\section{Device of photo-catalysis we designed}

As shown as figure 2, the gas fume primary treated entrance the device to dispose VOCs. Under the irradiation of ultraviolet light, through nano photo-catalytic net, catalytic oxidation of VOCs. 


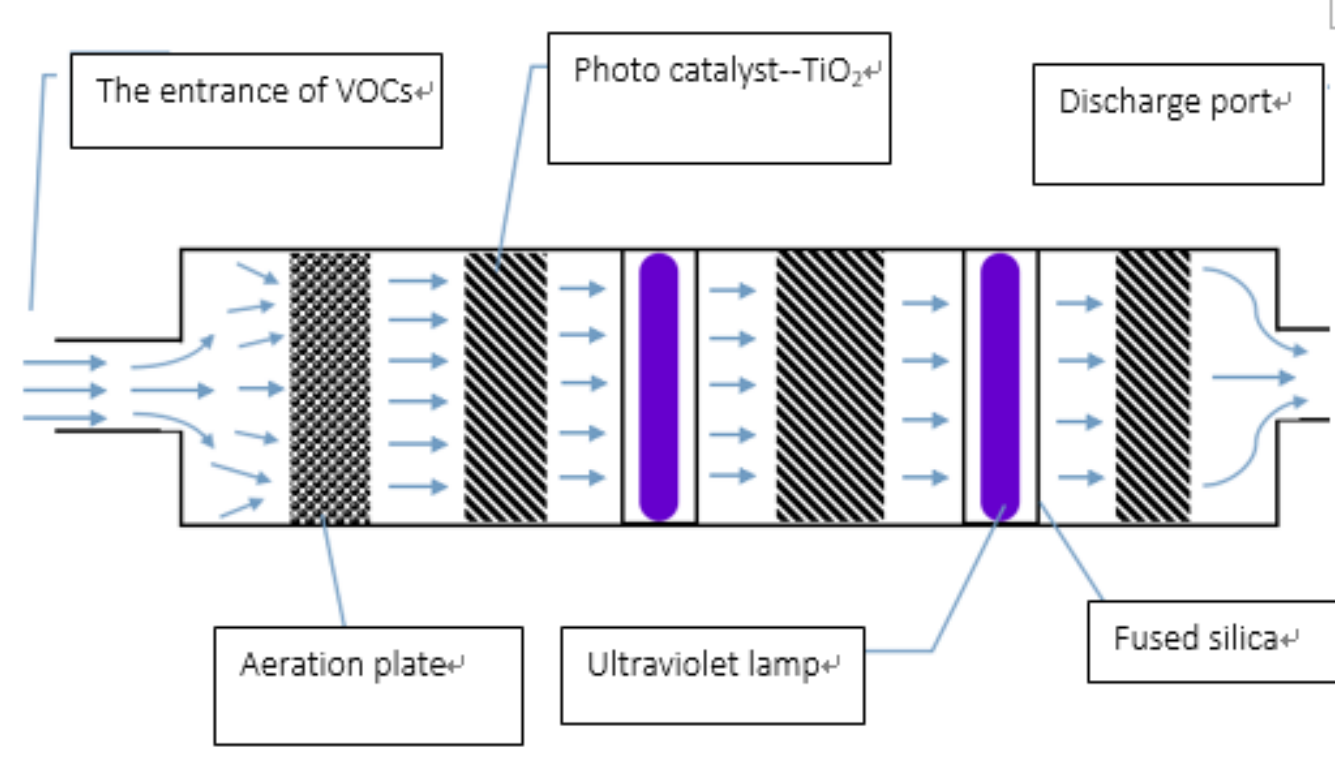

Figure 2 Device of photo-catalysis

\section{Status Quo and Development of Technique Standard}

Since the 1990s, Chinese relevant department pay more attention to the emission of the cooking fumes. However, the Degree of the Smell Reduction is to test the requirements of the inner environment brought by the emission ability of the kitchen ventilators. But it puts none limitation to the emission concentration of VOCs, PM10 or PM2.5. PM and VOCs are the major pollutant for air pollution and smog. Meanwhile, the contribution of the cooking fumes should not be underestimated. Believe it or not, It is necessary to impose the restriction like PM and VOCs to the emission of the kitchen ventilators.

\section{Conclusion}

VOCs from cooking fumes become the major factor to form smog. The treatment of the VOCs will be an irresistible trend. Hence, adding the VOCs photocatalytic degradation devices to the kitchen ventilators could control the emission of VOCs and conserve energy and reduce emission.

\section{Reference}

[1] Pang S W,Wong A. Challenges on the control of cooking fume emissions from restaurants [C]//Better Air Quality In Asian And Pacific Rim Cities, Hong Kong SAR ,16 Dec 2002-18 Dec 2002:1-7

[2] Zhao Y. Chemical compositions of fine particulate organic matter emitted from Chinese cooking [J].Environ Sci Technol, 2007, 41:99-105

[3] Huang D W, The impaction of cooking PM2.5 [ J ] .Environment, 2013, (11):69-71

[4] Abdullahi K L,Delgado-Saborit J M,Harrison R M. Emissions and indoor concentrations of particulate matter and its specific chemical.compnents from cooking: A review [J] ,Atmospheric Environment,2013,71:260-290

[5] Hisahiro E, Shigreru F,Takashi I, applied Catalysis B:Encironmental,38(2002)215

[6] Rafael M.R,Nelson C.M,Catal.Today,40(1998)353 